Please do not remove this page

RMIT

UNIVERSITY

\title{
Impact of nanogold morphology on interactions with human serum
}

Carnovale, Catherine; Bryant, Gary; Shukla, Ravi; Bansal, Vipul

https://researchrepository.rmit.edu.au/esploro/outputs/9921863346001341/filesAndLinks?institution=61RMIT_INST\&index=null

Carnovale, C., Bryant, G., Shukla, R., \& Bansal, V. (2018). Impact of nanogold morphology on interactions with human serum. Physical Chemistry Chemical Physics, 20(46), 29558-29565.

https://doi.org/10.1039/C8CP05938A

Document Version: Accepted Manuscript

Published Version: https://doi.org/10.1039/C8CP05938A

Repository homepage: https://researchrepository.rmit.edu.au

This journal is (c) the Owner Societies 2018

Downloaded On 2023/04/26 19:58:17 +1000

Please do not remove this page 


\title{
Impact of nanogold morphology on interactions with human serum
}

\section{Carnovale, ${ }^{\mathrm{a}, \mathrm{b}}$ G. Bryant ${ }^{\mathrm{b}}$ R. Shukla ${ }^{\mathrm{a}}$ and V. Bansal ${ }^{\mathrm{a}}$}

a. Ian Potter NanoBioSensing Facility, School of Science, RMIT University, GPO Box 2476, Melbourne VIC 3001 Australia. E-mail: vipul.bansal@rmit.edu.au

b. Centre for Molecular and Nanoscale Physics, School of Science, RMIT University, GPO Box 2476, Melbourne VIC 3001, Australia

\begin{abstract}
Gold nanoparticles (AuNPs) of differing shapes are of great interest to researchers due to their unique optical properties, making them potentially powerful theranostic tools. The synthesis of AuNPs is performed frequently, however the assessment of biological activity for each nanoparticle is not always commonplace. While it is thought that physicochemical parameters such as shape may play an important role in dictating the outcomes of interactions which take place at the nano-bio interface, a systematic approach to the assessment of nanomaterials has not been widely adopted. In this study, the interaction between human serum albumin (HSA) and four similar sized but different shaped AuNPs (spherical, rod shaped, prismatic and cubic) synthesised using a common chemical surfactant (CTAB), is presented. Using fluorescence spectroscopy it was shown that all AuNPs exhibit static binding with HSA, however the shape affects both the affinity and strength of the binding. Rod shaped nanoparticles were found to have the highest binding strength and affinity. Conversely, shapes with large flat planar surfaces such as prisms and cubes were shown to have reduced accessibility to the site of the fluorophore within the structure of HSA. The differences observed help to provide a better understanding of the effect of shape on AuNP-protein interactions - knowledge which may be applied to the development of AuNPs for future biological applications.
\end{abstract}




\section{Introduction}

The most abundant protein found in human blood, ${ }^{1}$ human serum albumin (HSA) plays a variety of physiological roles, including the regulation of blood $\mathrm{pH}$ and colloid osmotic pressure and the transport and circulation of various compounds including fatty acids, hormones and drugs. ${ }^{1,2}$ Preservation of certain parts of the complex structure of proteins such as HSA is vital to ensure that it continues to perform these functions. ${ }^{3}$ However, the exposure of proteins to various ligands and nanoparticles such as nanogold (AuNPs) is known to induce conformational changes. ${ }^{4,5}$ These changes may alter or inhibit the function of the protein which could potentially lead to serious health consequences.

It is well known that different morphologies of AuNPs elicit different biological responses. ${ }^{6,7}$ However it remains unclear if the effects observed to date are primarily due to differences in geometry, or differences in other factors such as chemical nature, surface charge or a combination of these. ${ }^{8}$ Due to their geometry, interesting optical properties emerge from nanoparticles with altered morphologies, providing potential myriad uses with diagnostic and therapeutic potential. ${ }^{9,} 10$ While there are a vast number of synthesis methods, biological response data is rather limited for these materials, particularly for cubic (AuNC) and prismatic (AuNPr) gold nanoparticles. The effect of gold nanoparticle shape has been the subject of numerous studies, most commonly focused on relating shape to toxicity and uptake. ${ }^{11-13}$ This leaves the relationship between nanoparticle shape and protein corona formation relatively unexplored, despite the likelihood that it may underpin the effects we see with regards to cellular response.

While the interaction between different shaped nanoparticles and HSA has been explored computationally, ${ }^{14}$ a study of this nature has yet to be performed in vitro. Surprisingly, to the best of the authors' knowledge, there is no study in which AuNPs of different morphologies, yet produced using a similar group of chemicals, have been tested in a complex biomimetic environment.

To address this gap, in this study we synthesise AuNPs of diverse morphologies (spherical, cubic, rod, and prismatic) using similar chemical routes and cetyltrimethylammonium bromide (CTAB) as a common surfactant. ${ }^{15}$ While the synthesis of other shapes (hollow 'nanorattles' ${ }^{16}$ or nanostars ${ }^{17}$ for example) is technically possible, the four shapes synthesized in this study were chosen explicitly as their synthesis could be performed using a common surfactant (CTAB).

CTAB, a cationic surfactant, is commonly incorporated into antiseptic formulations, and is often documented to be toxic to cells in its free form. Various research groups have shown that with careful preparation, nanoparticles that utilise CTAB for shape direction do not necessarily exhibit the same level of toxicity as observed with free CTAB. ${ }^{18}$ 
We subsequently employ fluorescence spectroscopy to study the interaction of each AuNP with HSA, as this technique provides a convenient tool to study the changes that proteins undergo on exposure to nanoparticles. ${ }^{19-}$ ${ }^{21}$ This is because proteins typically possess intrinsic fluorescent properties due to the presence of aromatic amino acid residues (tryptophan, tyrosine and phenylalanine) in their structure. ${ }^{22}$

The structure of HSA (PDB ID: 1AO6) shown in Figure 1 was downloaded from the Protein Data Bank. ${ }^{23}$ Multiple conformational forms of HSA are known to exist, dependent on the $\mathrm{pH}$ of the surrounding medium. The $\mathrm{N}$, or normal form of HSA is the expected conformation within these experiments which were carried out within a pH range of 7-8. ${ }^{24}$

This form of HSA is known to be comprised of three similarly arranged domains (I, II, and III) each consisting of two subdomains ( $A$ and $B$ ). Located within each subdomain is a possible site for binding and interaction.

Each of HSA's domains can be described as having a net overall charge.

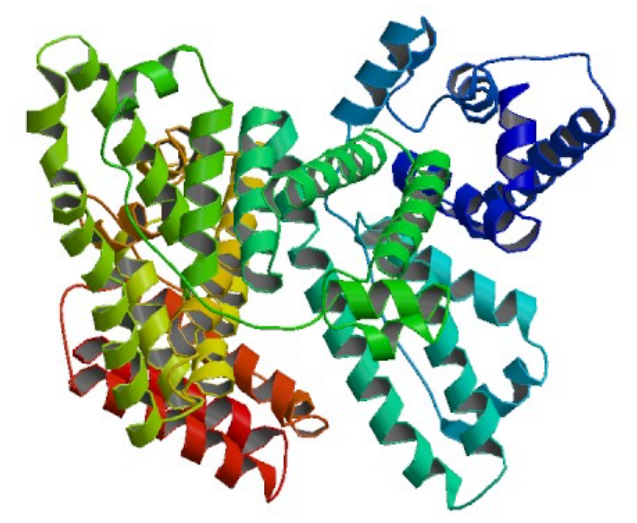

Fig 1: Crystal structure for HSA (PDB IS 1AO6) obtained from the Protein Data Bank. ${ }^{23}$

The III domain is the only to carry a net positive charge, $+2 \mathrm{e}$, while the I and II domain possess a net negative charge of $-9 \mathrm{e}$ and $-8 \mathrm{e}$ respectively. Due to the cationic nature of $C T A B$, the initial binding sites are proposed to be located within the I or II domain, and due to the similarity in charge little preferential affinity has been demonstrated between the two sites. ${ }^{25}$

HSA contains a single tryptophan residue which is contained in the hydrophobic cavity of sub domain IIA (Sudlow 1). ${ }^{26}$ The IIA sub domain of HSA has in fact been shown to play an important role in the binding of various ligands including AuNPs. ${ }^{27,}{ }^{28}$ Therefore, this single tryptophan residue in HSA becomes highly sensitive to changes in the surrounding environment and as such, provides a good opportunity to study the interaction between HSA and AuNPs. ${ }^{19}$ In particular, conformational disturbances in this important protein can then be detected by measuring changes in the emission features of the tryptophan residue during interaction of HSA with different morphologies of AuNPs. 


\section{Methods}

\section{Preparation of CTAB-stabilised gold nanospheres (AuNS)}

Preparation of AUNS was achieved by employing a modified method adapted from Rayavarapu et $a{ }^{29}$. In a typical synthesis a gold seed was created by combining $9.75 \mathrm{~mL}$ of $0.1 \mathrm{M} \mathrm{CTAB}$ with $250 \mu \mathrm{L}$ of $10 \mathrm{mM} \mathrm{HAuCl}_{4}$ while stirring. To this solution, $600 \mu \mathrm{L}$ of ice-cold freshly prepared $10 \mathrm{mM} \mathrm{NaBH}_{4}$ was injected while stirring vigorously. The solution was stirred for a further 2 minutes to allow excess $\mathrm{NaBH}_{4}$ to breakdown, with the resulting solution used within 5 minutes as a seed in the following reaction.

To prepare the growth solution, $38 \mathrm{~mL}$ of $0.1 \mathrm{M} \mathrm{CTAB}$ solution was prepared and brought to a temperature of 25 ${ }^{\circ} \mathrm{C}$, after which $2.0 \mathrm{~mL}$ of $10 \mathrm{mM} \mathrm{HAuCl}{ }_{4}$ and $210 \mu \mathrm{L}$ of $0.1 \mathrm{M} \mathrm{L}$-ascorbic acid were added, stirring after each addition. Lastly, $48 \mu \mathrm{L}$ of gold seed solution was injected into the growth solution and gently stirred before being left at $25^{\circ} \mathrm{C}$ for 24 hours.

Following this period, the solutions were washed to remove excess CTAB and potentially unreacted constituents. The solutions were first centrifuged at $500 \times g 2000$ for 6 minutes to settle excess CTAB, the supernatant collected and the process repeated while the pellet (made up of precipitated excess CTAB) was discarded. Following this, the supernatant was centrifuged at $4500 \times g 6000 \mathrm{RPM}$ for 12 minutes at $25^{\circ} \mathrm{C}$, repeated 3 times, each wash discarding the supernatant and redispersing the pellet in deionised (Milli-Q) water.

\section{Preparation of CTAB-stabilised gold nanorods (AUNR)}

A modified version of the method described by Liu and Guyot-Sionnest ${ }^{30}$ was employed to synthesise AuNR. In a typical synthesis gold seeds were created using a method devised by Nikoobakht and El-Sayed. ${ }^{31}$ To achieve this, $10 \mathrm{~mL}$ of $0.1 \mathrm{M} \mathrm{CTAB}$ was brought to $30{ }^{\circ} \mathrm{C}$, to which $250 \mu \mathrm{L}$ of $10 \mathrm{mM} \mathrm{HAuCl}_{4}$ was added while stirring. To this solution, $600 \mu \mathrm{L}$ of freshly prepared $10 \mathrm{mM} \mathrm{NaBH}_{4}$ was injected while stirring vigorously. The solution was stirred for a further 5 minutes to allow excess $\mathrm{NaBH}_{4}$ to breakdown, with the resulting solution used as a seed in the following reaction.

$40 \mathrm{~mL}$ of $0.1 \mathrm{M} \mathrm{CTAB}$ solution was freshly prepared and brought to a temperature of $30^{\circ} \mathrm{C}$, following which $2.0 \mathrm{~mL}$ of $10 \mathrm{mM} \mathrm{HAuCl}_{4}, 0.4 \mathrm{~mL}$ of $10 \mathrm{mM} \mathrm{AgNO}_{3}, 800 \mu \mathrm{L}$ of $0.1 \mathrm{M} \mathrm{HCl}$ and $320 \mu \mathrm{L}$ of $0.1 \mathrm{M} \mathrm{L}$-ascorbic acid were added, while stirring after each addition. Lastly, $96 \mu \mathrm{L}$ of gold seed solution was injected into the growth solution and gently stirred before being left for 2 hours at $30^{\circ} \mathrm{C}$.

The solution was allowed to stabilise at room temperature for 24 hours before being washed to remove excess $\mathrm{CTAB}$ and potentially unreacted constituents. The solution was first centrifuged at $500 \times g 2000$ for 6 minutes to precipitate and remove excess CTAB. The supernatant was then centrifuged at $8000 \times g 8000$ RPM for 15 minutes at $12{ }^{\circ} \mathrm{C}$, repeated 3 times, each wash discarding the supernatant and redispersing the pellet in deionised water. 


\section{Preparation of CTAB-stabilised gold nanoprisms (AuNPr)}

The method devised by Millstone et al. was employed to synthesise AuNPr. ${ }^{32}$ In a typical synthesis, $5 \mathrm{~nm}$ gold seeds were prepared by sequentially adding $1 \mathrm{~mL}$ of $0.01 \mathrm{M}$ sodium citrate dihydrate, $1 \mathrm{~mL}$ of $0.01 \mathrm{M} \mathrm{HAuCl}_{4}$ and $1 \mathrm{~mL}$ freshly prepared $0.1 \mathrm{M} \mathrm{NaBH}_{4}$ to $36 \mathrm{~mL}$ of deionised water while stirring vigorously. The gold seeds were allowed to mix for a further 1 minute before being left undisturbed for 2 hours and to be used in the following reaction.

An iodide doped CTAB mixture was prepared for the growth solutions by adding $2.733 \mathrm{~g}$ of purified CTAB in 150 $\mathrm{mL}$ of deionised water, followed by the addition of $75 \mu \mathrm{L}$ of $0.1 \mathrm{M} \mathrm{KI}$. The solution vial was sealed, gently heated until the CTAB dissolved, then sonicated for 30 seconds before being set aside at room temperature for 2 hours.

Following this, three growth solutions were prepared, such that growth solutions 1 and 2 were each made up of 9 $\mathrm{mL}$ of the prepared iodide doped CTAB solution, $250 \mu \mathrm{L}$ of $0.01 \mathrm{M} \mathrm{HAuCl}_{4}, 50 \mu \mathrm{L}$ of $0.1 \mathrm{M} \mathrm{NaOH}$ and $50 \mu \mathrm{L}$ of 0.1 M L-ascorbic acid, while solution 3 contained $90 \mathrm{~mL}$ of prepared iodide doped CTAB solution, $2.5 \mathrm{~mL}$ of $0.01 \mathrm{M}$ $\mathrm{HAuCl}_{4}, 500 \mu \mathrm{L}$ of $0.1 \mathrm{M} \mathrm{NaOH}$ and $500 \mu \mathrm{L}$ of $0.1 \mathrm{M} \mathrm{L}$-ascorbic acid.

To commence the reaction, $1 \mathrm{~mL}$ of the $5 \mathrm{~nm}$ seed solution was added to growth solution 1 which was gently stirred before transferring $1 \mathrm{~mL}$ of growth solution 1 into growth solution 2, which was stirred before adding its total contents into growth solution 3 . The reaction was allowed to proceed undisturbed for 30 minutes until completion.

The solution was left to stabilise for 24 hours before being washed to remove excess CTAB and unreacted constituents. The solutions were first centrifuged at $500 \times g 2000$ for 6 minutes to precipitate and remove excess CTAB. Following this, the supernatant was centrifuged at $8000 \times g 8000$ RPM for 3 minutes at room temperature. This process was repeated 3 times, discarding the supernatant and redispersing the pellet in deionised water after each wash.

\section{Preparation of CTAB-stabilised gold nanocubes (AuNC)}

A modified version of the method detailed by Kim et al. was employed to synthesise AuNC. ${ }^{33}$ In a typical synthesis $40 \mathrm{~mL}$ of $10 \mathrm{mM} \mathrm{CTAB}$ was made in a $50 \mathrm{~mL}$ tube to which $500 \mu \mathrm{L}$ of $10 \mathrm{mM} \mathrm{HauCl}_{4}$ was added. The solution was mixed by gentle inversion before $200 \mu \mathrm{L}$ of $100 \mathrm{mM}$ L-ascorbic acid was added, followed by repeated inversion to mix.

The solution was allowed to stand for 30 minutes to allow the ascorbic acid to begin the reduction of $\mathrm{Au}^{3+}$ to $\mathrm{Au}^{+}$. After this period, $60 \mu \mathrm{L}$ of $100 \mathrm{mM} \mathrm{NaOH}$ was slowly added to the bottom of each tube using a pipette, which was removed carefully with minimal disturbance to the solution. The solution was allowed to stand at $25{ }^{\circ} \mathrm{C}$ for 6 hours before the tubes were inverted. By this time, the addition of $\mathrm{NaOH}$ had further reduced $\mathrm{Au}^{+}$to $\mathrm{Au}^{0}$, which seeded the growth of cubic shaped gold nanoparticles. 
The solution was allowed to stabilise for 24 hours before being washed to remove excess CTAB and unreacted constituents. The solutions were first centrifuged at $500 \times g 2000$ for 6 minutes at room temperature to precipitate and discard excess CTAB. Following this, the solutions were centrifuged at $10000 \times g 9000$ for 3 minutes, repeated 3 times, discarding the supernatant and redispersing the pellet in deionised water after each wash.

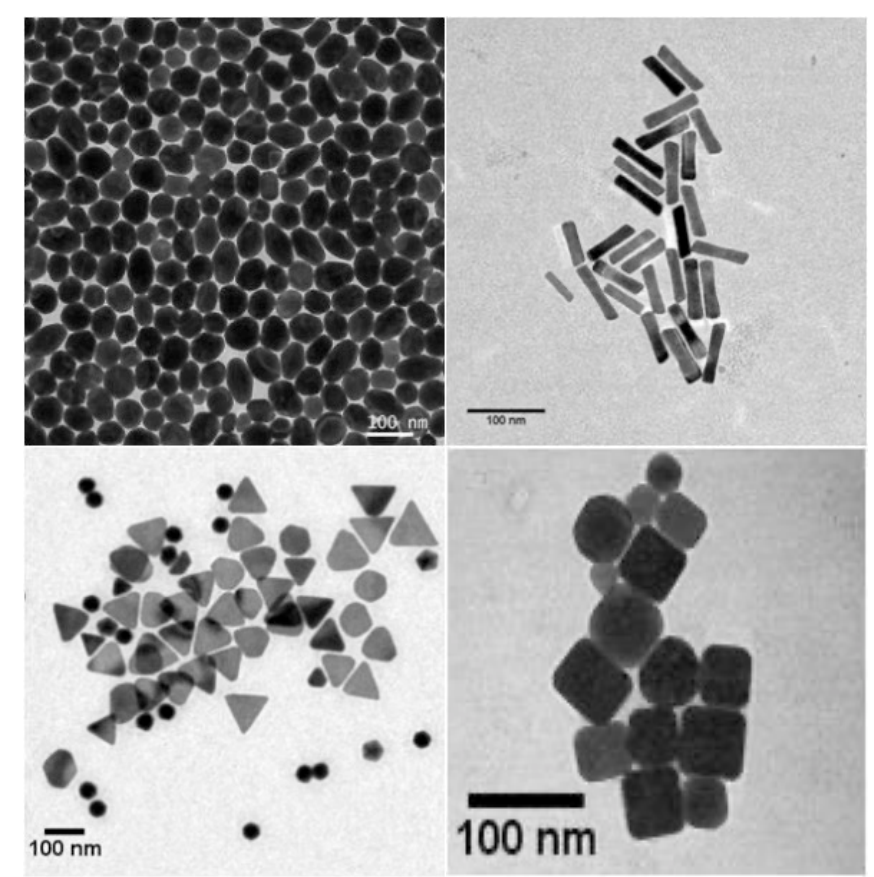

Fig 2: TEM images (clockwise from top left) AuNS, AuNR, AuNPr, and AuNC.

\section{Fluorescence spectroscopy}

To study the interaction of AuNPs with HSA, fluorescence measurements were performed using a Horiba Fluormax 4 - Compact Spectrofluorometer which utilises a 150W Xenon arc lamp, Czerny-Turner monochromator and a R928P photon counting PMT detector with internal temperature control. It is well-known that when the tryptophan fluorophore in HSA is photoexcited at $295 \mathrm{~nm}$ wavelength ${ }^{34}$, it produces an emission peak between 300 and $500 \mathrm{~nm}$, so fluorescence spectra were generated under these conditions.

A series of AuNPs concentration (2.5, 25, 50, 100 and $250 \mu \mathrm{M})$ and reaction temperature $(20,30,40,50$ and 60 ${ }^{\circ} \mathrm{C}$ ) dependent emission spectra were obtained by incubating the AuNPs for 5 minutes with a fixed (final) concentration of HSA (200 $\mathrm{\mu g} / \mathrm{mL})$. The indicated AuNP concentrations correspond to the equivalent of $\mathrm{Au}$ ion concentrations in nanoparticles, as determined by atomic absorption spectroscopy after digesting the AuNPs in aqua regia. The 5 minute time point for these studies was determined following a time optimisation study, and is sufficient to allow AuNP-HSA binding to occur and allow reproducible measurements after temperature stabilisation. 


\section{Dynamic Light Scattering}

Dynamic Light Scattering (DLS) was conducted on an ALV-5022F light scattering spectrometer with a $632.8 \mathrm{~nm}$ $\mathrm{He}-\mathrm{Ne}$ laser at a scattering angle of $90^{\circ}$. Samples were measured in $10 \mathrm{~mm}$ cylindrical cuvettes maintained at a fixed temperature of $22^{\circ} \mathrm{C}$, and data was averaged for $120 \mathrm{~s}$. Average particle size was determined using the cumulant fit in the in-built software.

\section{Results and discussion}

TEM images of each AuNP are presented in Fig 2. All samples appear not to be aggregated once dried. At least 100 particles were analysed to determine average particle dimensions (diameter for AuNS and average side length for AuNR, AuNPr and AuNC). These measurements, as well as the standard deviation (SD) for each value, are shown in Table 1 alongside the hydrodynamic diameters determined using Dynamic Light Scattering (DLS). Zeta potential measurements for all particles are presented, where, as expected, all particles synthesised with the use of CTAB had positive zeta potentials, regardless of shape.

\begin{tabular}{|l|l|l|l|}
\hline Shape & $\begin{array}{l}\text { Hydrodynamic } \\
\text { Diameter (DLS) }\end{array}$ & $\begin{array}{l}\text { TEM - Average } \\
\text { measurement } \pm \text { SD }\end{array}$ & Zeta Potential \\
\hline Sphere & $80 \mathrm{~nm}$ & $\begin{array}{l}77.9 \pm 3.5 \mathrm{~nm} \\
\text { (diameter) }\end{array}$ & +44.9 \\
\hline Rod & $\mathrm{n} / \mathrm{a}$ & $\begin{array}{l}58.8 \pm 10.6 \mathrm{~nm} \\
\text { (length) }\end{array}$ & +38.1 \\
\hline Prism & $\begin{array}{l}5.3 \pm 5.1 \mathrm{~nm} \\
\text { (width) }\end{array}$ & \\
\hline Cube & $90 \mathrm{~nm}$ & $\begin{array}{l}94.7 \pm 15.6 \mathrm{~nm} \\
\text { (side length) }\end{array}$ & +52.2 \\
\hline & $115 \mathrm{~nm}$ & $\begin{array}{l}47.5 \pm 11.8 \mathrm{~nm} \\
\text { (side length) }\end{array}$ & +30.4 \\
\hline
\end{tabular}

Table 1: Physicochemical parameters for all particles.

While spherical AuNPs characteristically give a single absorption peak in the UV-visible spectrum, various shapes with inherent anisotropy such as rods, prisms, stars and branches can give rise to two or more absorption peaks as their complex geometry creates varied paths along which the surface plasmons oscillate.

The characteristic UV-visible absorption spectrum for AUNS spherical particles with a single absorption band at $540 \mathrm{~nm}$ shown in Fig. 3. The AuNR returned a typical spectrum for nanorods of high purity (low concentration of spheres) evidenced by the weakness of the transverse peak at $510 \mathrm{~nm}$ in relation to the longitudinal peak at 875 
$\mathrm{nm} \cdot{ }^{35}$ Similarly, prismatic gold nanoparticles are often characterised by having multiple absorption peaks - with their positions dependant on the edge lengths of the particles. ${ }^{36}$

The AuNPr presented in Fig. $\mathbf{2}$ show the transverse peak at $550 \mathrm{~nm}$ with the longitudinal peak seen as a shoulder at $630 \mathrm{~nm}$. The symmetry associated with cubic structures dictates that cubic AuNPs have a single absorption peak, whose position, like spherical particles, is dependent on the size of the particles. ${ }^{37} \mathrm{~A}$ red shift is expected as the side length increases which is consistent with the spectrum obtained for the AuNC presented here with a single peak at $554 \mathrm{~nm}$.
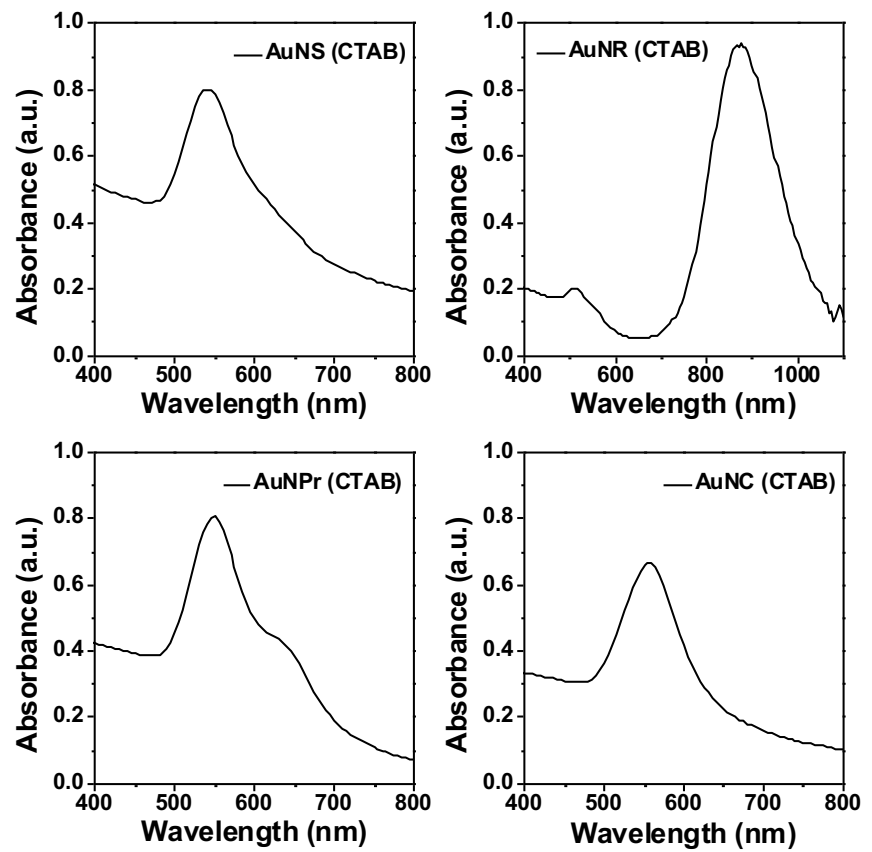

Fig 3: UV-visible absorption spectra (clockwise from top left) AuNS, AuNR, AuNPr and AuNC.

The relationship between fluorescence quenching $(Q)$ and fluorescence intensity $(F)$ can be assessed using Equation $1 .{ }^{19}$

$$
Q=\left(\frac{F_{0}-F}{F_{0}}\right) \quad \text { Equation } 1
$$

To quantify this relationship, fluorescence measurements were performed on HSA in the absence $\left(F_{0}\right)$ and presence $(F)$ of AuNPs. While the final concentration of HSA remained the same in each experiment $(200 \mu \mathrm{g} / \mathrm{mL})$, the concentration of AuNPs was varied from 2.5 to $250 \mu \mathrm{M}$.

The emission spectra of HSA in Fig. 4 demonstrate that the fluorescence of the HSA molecule quenches in the presence of AuNPs regardless of morphology. The fluorescence quenching consistently increases with increasing AuNP concentration. Also shown are curves for the highest tested concentration of AuNPs (250 mM) without HSA, to demonstrate that the AuNPs are not inherently fluorescent. 
In general, there are two main mechanisms by which fluorescence quenching occurs; viz. dynamic or static. ${ }^{38}$ Dynamic quenching stems from collisions between the fluorophore (HSA) and the fluorescence quencher (AuNP) and results in a loss of excited state energy. In the case of dynamic quenching, the collisions must occur while the fluorophore is in an excited state. On the other hand, static quenching occurs as a result of a non-fluorescent complex forming between the fluorophore and the quencher, a process which may occur before the fluorophore is excited.
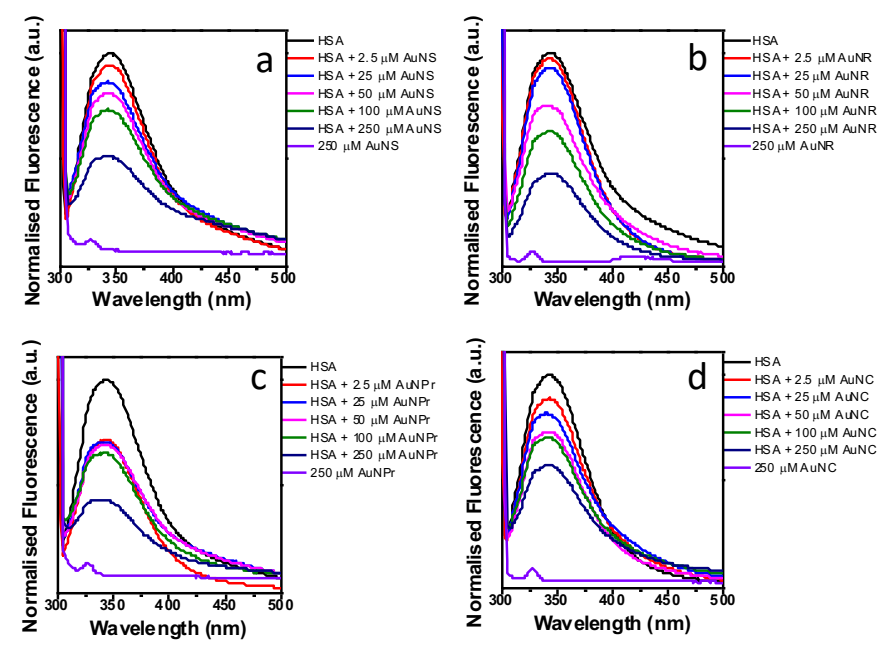

Fig. 4: Fluorescence quenching of HSA by (a) AuNS, (b) AuNR, (c) AuNPr, and (d) AuNC. Emission spectra were collected at $20^{\circ} \mathrm{C}$.

Interactions can be categorised as being either static or dynamic, or a combination of both by employing the Stern-Volmer equation (Equation 2). ${ }^{39}$

$$
\frac{F_{0}}{F}=1=k_{q} \tau_{0}[Q]=1+K_{S V}[Q] \quad \text { Equation } 2
$$

Where $k_{q}$ is the biomolecular quenching rate constant, $\tau_{0}$ is the lifetime of the fluorophore (approximately equal to $4.9 \mathrm{~ns}$ for $\mathrm{HSA}),{ }^{40}[Q]$ is the concentration of AuNPs, and $K_{S V}$ is the Stern-Volmer constant.

By plotting $\frac{F_{0}}{F}$ versus [Q], Stern-Volmer plots were generated for all AuNPs as shown in Fig. 5. The Stern-Volmer constant is calculated as the slope of the linear regression fit of the data points.

Linear agreement to the equation usually signifies either static or dynamic quenching while positive deviation signifies a combination of both mechanisms. Fig. $\mathbf{5}$ shows that AuNS, AuNR and AuNC show linear agreement across all concentrations measured, while AuNPr shows a linear fit up to a concentration of $100 \mu \mathrm{M}$.

To clearly identify these mechanisms, multiple temperature measurements are required to differentiate between static and dynamic quenching. ${ }^{38}$ The full concentration range was used to determine the quenching mechanism 
for AuNS, AuNR and AuNC, whereas the linear range $(0-100 \mu \mathrm{M})$ was used to determine the quenching mechanism for AuNPr.
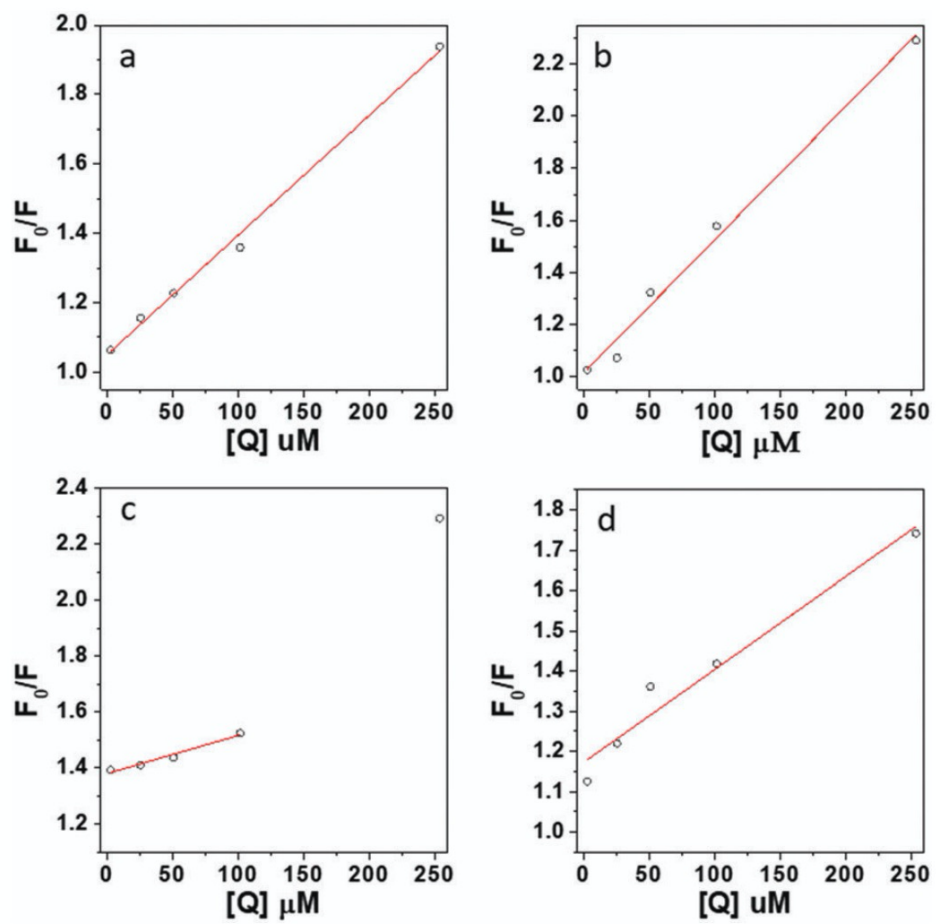

Fig. 5: Stern-Volmer plots obtained for (a) AuNS, (b) AuNR, (c) AuNPr, and (d) AuNC at $20^{\circ} \mathrm{C}$.

In the case of dynamic quenching which relies on collisions, $K_{S V}$ is expected to increase with temperature as the diffusion rate increases. On the other hand, static quenching is inversely related to temperature with stronger complexes formed at lower temperatures. To determine the method of binding of HSA with each of the morphologies of AuNPs, Stern-Volmer plots were generated over a range of temperatures from $20-60{ }^{\circ} \mathrm{C}$. Figure 6 illustrates one such Stern-Volmer plot for AuNS at temperatures ranging from $20-60{ }^{\circ} \mathrm{C}$ while the results for all AuNPs are summarised in Table 2. The $K_{S V}$ values presented in Table 1 all decrease with increasing temperature, establishing that all four tested morphologies of AuNPs exhibit static interactions with HSA molecules.

For static quenching, $K_{s V}$ values provide an indication of the association constant for the newly formed complex between the fluorophore and the quencher. ${ }^{1,41}$ The comparison of the $K_{S V}$ values for different morphologies of AuNPs suggests that AuNR have the highest level of interaction with HSA, and as such, the strength of binding between the nanoparticles and HSA follows the order AuNR $>$ AuNS $>$ AuNC $>$ AuNPr. 


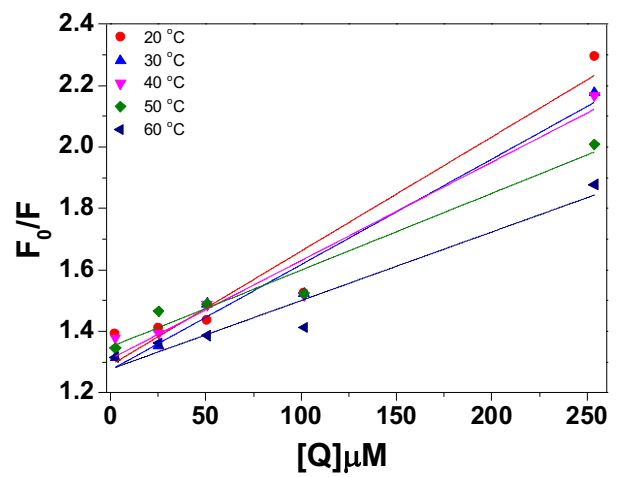

Fig. 6: Stern-Volmer plots for HSA quenching by AuNS at different temperatures. Measurements at 20-60 ${ }^{\circ} \mathrm{C}$ show decreased $K_{S V}$ with increasing temperature.

\begin{tabular}{|c|c|c|c|c|}
\hline & \multicolumn{2}{|c|}{ AuNS } & \multicolumn{2}{|c|}{ AuNR } \\
\hline Temperature & $\kappa s v$ & $\mathbf{R}$ & $K s v$ & $\mathbf{R}$ \\
\hline $20^{\circ} \mathrm{C}$ & 3.5 & 0.997 & 5.1 & 0.995 \\
\hline $30{ }^{\circ} \mathrm{C}$ & 3.5 & 0.997 & 4.9 & 0.996 \\
\hline $40^{\circ} \mathrm{C}$ & 3.3 & 0.997 & 4.7 & 0.995 \\
\hline $50^{\circ} \mathrm{C}$ & 3.3 & 0.997 & 4.5 & 0.994 \\
\hline \multirow[t]{2}{*}{$60^{\circ} \mathrm{C}$} & 2.9 & 0.997 & 4.3 & 0.998 \\
\hline & \multicolumn{2}{|c|}{ AuNPr } & \multicolumn{2}{|c|}{ AuNC } \\
\hline Temperature & $K_{s v}$ & $\mathbf{R}$ & $K_{s v}$ & $\mathbf{R}$ \\
\hline $20^{\circ} \mathrm{C}$ & 2.1 & 0.992 & 2.3 & 0.980 \\
\hline $30{ }^{\circ} \mathrm{C}$ & 2.2 & 0.926 & 2.1 & 0.989 \\
\hline $40^{\circ} \mathrm{C}$ & 1.5 & 0.933 & 1.9 & 0.985 \\
\hline $50^{\circ} \mathrm{C}$ & 1.6 & 0.861 & 1.6 & 0.983 \\
\hline $60{ }^{\circ} \mathrm{C}$ & 0.93 & 0.949 & 1.9 & 0.985 \\
\hline
\end{tabular}

Table 2: Calculated Stern-Volmer constants for the quenching of HSA by different morphologies of AuNPs across the temperature range of $20-60{ }^{\circ} \mathrm{C} . \mathrm{R}$ is the linear correlated coefficient for $K_{S V}$ values. $K_{S V}$ values are expressed as $\times 10^{3} \mathrm{~L} \mathrm{~mol}^{-1}$.

For static quenching, $K_{S V}$ values provide an indication of the association constant for the newly formed complex between the fluorophore and the quencher. ${ }^{1,41}$ The comparison of the $K_{S V}$ values for different morphologies of AuNPs suggests that AuNR have the highest level of interaction with HSA, and as such, the strength of binding between the nanoparticles and HSA follows the order AuNR > AuNS $>$ AuNC $>$ AuNPr. 
The highest affinity of HSA to AuNR may be attributed to the preferential binding of CTAB molecules to different crystal planes of a nanorod during crystal growth. The shape-directing ability of CTAB to form nanorods is believed to be due to the ability of CTAB to bind strongly on the longitudinal faces of nanorods forming a bilayer. In contrast, the short ends of nanorods are believed to be free from CTAB, allowing the nanorods to preferentially grow in one direction. ${ }^{21,42}$ This may offer serum proteins an opportunity to preferentially bind to the surfactantfree ends of CTAB-stabilised AuNR. In our case, the transverse ends of AuNR are approximately $15 \mathrm{~nm}$ wide (Table 3), which should be large enough to interact with HSA with high affinity. It is also noted that while there are limited studies in this area, a previous study found that the interaction of the bovine serum albumin protein was stronger with AuNR than AuNPr. ${ }^{43}$ Our current study further strengthens the argument that the morphology of nanomaterials plays an important role in their interaction with proteins.

It is also worth noting that while our current study compares AuNPs of different morphologies at the same concentrations, these concentrations represent Molar equivalent of gold atoms in the sample. However, due to the difference in the morphologies and dimensions of the AuNPs, the total number of particles and the corresponding surface area available to interact with a fixed amount of HSA molecules will be different in each case. This was also noted previously, where smaller silver nanoparticles were found to have stronger interactions and more efficient binding with HSA. ${ }^{44}$ Table 3 provides a relative comparison of number of particles and available surface area for different morphologies of AuNPs, together with the biomolecular quenching rates for the HSA interaction. Table 3 highlights the lack of correlation between the available surface area and $K_{s v}$, suggesting it unlikely that the effects seen in this study are solely due to the surface area or number of particles. This suggests that other physico-chemical characteristics of nanomaterials play an important role in proteinnanomaterial interactions. These may, for instance, include the different chemical nature of different crystal planes (e.g. nanorods have more CTAB along their length over their sides), or even the potential ability of proteins to selectively interact with different crystal facets of a nanocrystal.

To further probe the binding action of each AuNP to HSA, the binding constant $k_{b}$ and the number of binding sites per HSA molecule were calculated using Equation $3 .{ }^{44}$

$$
\log \frac{F_{0}-F}{F}=\log k_{b}+n \log [Q] \quad \text { Equation } 3
$$

Figure 7 displays plots for $\log \frac{F_{0}-F}{F}$ versus $\log [Q]$ for each AuNP whereby the slope and intercept of each plot represent the number of binding sites $(n)$ and $\log k_{b}$ respectively. Linear fits were applied to AuNS, AuNR and AuNC data. However the AuNPr data showed two clear linear regions, so each was fit separately. Results for all AuNPs are summarised in Table 4. 
In general, for the majority of AuNPs examined, the number of binding sites, $n$, is of order 1 , indicating that there is one unique HSA binding site for each AuNP. However, the number of binding sites can also be considered as a measure of association cooperativity. ${ }^{19}$

For $n>1$, once a nanoparticle binds to a protein molecule, the protein increases its affinity for the bound nanoparticle, developing a stronger bond over time. For $n<<1$ (as noted for AuNS, AuNC and AuNPr) the opposite is true - AuNPs bound to an HSA molecule will have reduced affinity for additional HSA molecules.

\begin{tabular}{|c|c|c|c|c|c|}
\hline $\begin{array}{l}\text { AuNP } \\
\text { type }\end{array}$ & $\begin{array}{c}{ }^{1} \text { Average } \\
\text { AuNP } \\
\text { dimensions } \\
\text { (nm) }\end{array}$ & $\begin{array}{c}\begin{array}{c}{ }^{2} \text { Average } \\
\text { mass per } \\
\text { AuNP }\end{array} \\
{\left[m_{\text {AuNP }}\right]} \\
\left({ }^{*} 10^{15} \mathrm{~g}\right)\end{array}$ & $\begin{array}{c}{ }^{3} \text { Number } \\
\text { of AuNPs } \\
\text { per Mole } \\
\text { of gold } \\
\left(*^{*} 10^{-16}\right)\end{array}$ & $\begin{array}{l}{ }^{4} \text { Surface } \\
\text { area of } \\
\text { AuNPs } \\
\text { per Mole } \\
\text { of gold } \\
\left({ }^{*} 10^{-2} \mathrm{~m}^{2}\right)\end{array}$ & $\begin{array}{c}{ }^{5} \mathrm{Ksv} \\
\text { at } 20^{\circ} \mathrm{C} \\
\left({ }^{*} 10^{3} \mathrm{~L}\right. \\
\left.\mathrm{mol}^{-1}\right)\end{array}$ \\
\hline AuNR & $\begin{array}{l}58.8 \text { (length) } \\
15.3 \text { (width) }\end{array}$ & 0.3 & 74 & 30 & 5.1 \\
\hline AuNS & $\begin{array}{c}77.9 \\
\text { (diameter) }\end{array}$ & 4.8 & 4.1 & 7.9 & 3.4 \\
\hline AuNC & $\begin{array}{c}47.5 \\
\text { (edge length) }\end{array}$ & 2.1 & 9.5 & 13 & 2.3 \\
\hline $\begin{array}{c}\text { AuNPr } \\
*\end{array}$ & $\begin{array}{c}94.7 \\
\text { (edge length) } \\
25.0 \text { (height) }\end{array}$ & 1.9 & 11 & 16 & 2.1 \\
\hline
\end{tabular}

Table 3: Calculated parameters for different morphologies of AuNPs. ${ }^{1}$ Calculated by measuring at least 100 particles from TEM images; ${ }^{2}$ calculated by considering the density of gold as $19320 \mathrm{~kg} / \mathrm{m}^{3} ;{ }^{45}{ }^{3}$ calculated using the molecular weight of gold as $196.96 \mathrm{~g} / \mathrm{mol} ;{ }^{45}{ }^{4}$ calculated by $4 * \pi^{*} R^{2}$ for AuNS, $2 *\left(w^{2}\right)+4 *(I * w)$ for AuNR, $b * \mid+(3 * b)$

${ }^{*} \mathrm{~h}$ for AuNPr and $\left(\mathrm{I}^{2}\right)^{*} 6$ for AuNC; ${ }^{5}$ calculated through Stern-Volmer plots; *assumed height of AuNPr based on literature values. ${ }^{36}$

Therefore values less than one imply that the AuNP may only be partially bound to the HSA molecule. ${ }^{1}$ This is particularly evident in the case of near-zero $\log [Q]$ value for AuNPr for low nanoparticle concentrations. For $n \cong 1$ (as noted for AuNR) the binding affinity of AuNPs to HSA remains independent of the presence of other HSA molecules. ${ }^{44}$

Furthermore, the binding constant $k_{b}$ and number of binding sites $n$ are intrinsically linked, with higher $k_{b}$ values observed where increased affinity is observed ${ }^{46}$ Commonly, $k_{b}$ values follow similar trends to the $K_{s V}$ values. This holds true in our current study (AuNR $>$ AuNS $>$ AuNC $>$ AuNPr), with a notable observation that depending on the 
concentration of AuNPr, its binding affinity towards HSA may vary, such that increasing concentrations of AuNPr with a fixed amount of HSA greatly improves its binding affinity.
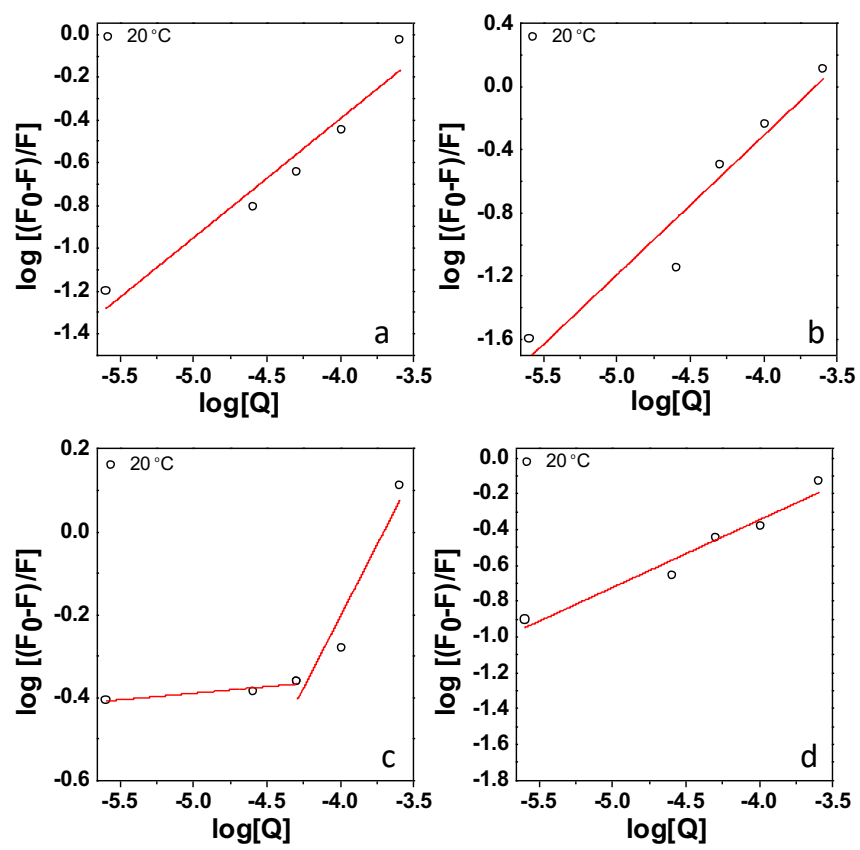

Fig. 7: Determination of the binding constant $k b$ and number of binding sites per HSA molecule for (a) AuNS, (b) AuNR, (c) AuNPr, and (d) AuNC.

\begin{tabular}{|c|c|c|c|c|c|}
\hline & AuNS & AuNR & \multicolumn{2}{|c|}{ AuNPr } & AuNC \\
\hline $\mathrm{kb}\left(\mathrm{L} \mathrm{mo}^{-1}\right)$ & 69 & 1702 & 0.58 & 365 & 15 \\
\hline $\mathrm{n}$ & 0.55 & 0.88 & 0.03 & 0.69 & 0.37 \\
\hline $\mathrm{R}$ & 0.97 & 0.97 & 0.93 & 0.96 & 0.98 \\
\hline$\Delta \mathrm{G}\left(\mathrm{kJ} \mathrm{mol}^{-1}\right)$ & -10 & -18 & 1.3 & -14 & -6.5 \\
\hline
\end{tabular}

Table 4: Binding constant $\left(k_{b}\right)$ and number of binding sites per HSA molecule $(n)$ for different morphologies of AuNPs as determined by linear fits of plots of $\log \frac{F_{0}-F}{F}$ versus $\log [Q]$. R is the linear correlated coefficient for $k_{b}$ and $n$ values. Change in free energy, $\Delta G$ resulting from binding of HSA to AuNPs, as obtained from Equation 4. Measurements were performed at $20^{\circ} \mathrm{C}$.

Furthermore, the binding constant $k_{b}$ and number of binding sites $n$ are intrinsically linked, with higher $k_{b}$ values observed where increased affinity is observed. ${ }^{46}$ Commonly, $k_{b}$ values follow similar trends to the $K_{s V}$ values. This 
holds true in our current study (AuNR $>$ AuNS $>$ AuNC $>$ AuNPr), with a notable observation that depending on the concentration of AuNPr, its binding affinity towards HSA may vary, such that increasing concentrations of AuNPr with a fixed amount of HSA greatly improves its binding affinity.

We further examined the thermodynamics of the reaction between AuNPs and HSA using Equation 4:

$$
\Delta G=-R T \ln k_{b} \quad \text { Equation } 4
$$

Where $\Delta G$ is the change in the free energy after binding of AuNPs to HSA, $R$ is the gas constant, $T$ is the temperature in Kelvin and $k_{b}$ is the binding constant. The results are compiled in Table 5.

While positive $\Delta G$ values indicate reactions requiring energy to proceed (endothermic reactions), negative $\Delta G$ values represent spontaneous reactions. All of the interactions between different morphologies of AuNPs and HSA are deemed to be spontaneous (with the exception of low concentrations of AuNPr), implying that energy is released during binding of AuNPs to HSA, leading to the transition towards a more stable, lower energy state. ${ }^{47}$ This in consistent with other studies investigating the thermodynamics of AuNPs with serum proteins. ${ }^{48,49}$ In the case of AuNPr, the low $k_{b}, \mathrm{n}$ and $\Delta G$ values indicate that binding with HSA does not proceed to a great degree at low concentrations. From these results it can be determined that AuNR release the greatest amount of energy upon binding to HSA while AuNC release approximately three times less energy per mole.

\begin{tabular}{|c|c|}
\hline AuNP & $\Delta G \mathrm{~kJ} \mathrm{~mol}^{-1}$ \\
\hline AuNS & -10.3 \\
\hline AuNR & -18.1 \\
\hline AuNPr & -10.1 \\
\hline AuNC & -6.5 \\
\hline
\end{tabular}

Table 5: Change in free energy, $\Delta G$ which occurs after binding of HSA to AuNPs at $20^{\circ} \mathrm{C}$.

\section{Conclusions}

It can be concluded from these results that shape plays an important factor in AuNP-protein interactions. The results indicate that HSA will bind with greater efficiency and affinity to rod shaped AuNPs. Additionally, AuNRs obtained the highest $K_{s v}$ values while shapes with large flat faces, such as prisms and cubes returned the lowest values. This is likely to be related not only to shape, but also to the dimensions of the specific AuNRs, as the small $(15 \mathrm{~nm})$ transverse dimension of the rod shaped particles may have greater ease of accessing the site of the fluorophore within HSA and may have less steric inhibition in binding compared with larger particle surfaces. 
Furthermore, the efficiency and affinity results were consistent with this, indicating that HSA binds least to AuNC and AuNPr. While this trend is likely related to AuNP shape, the exact mechanism remains unknown.

In the case of AuNPrs it is evident that the relationship between AuNP concentration and binding behaviour is not linear over the whole concentration range examined. This indicates that binding activity may follow different regimes depending on the concentration. Further modelling \& experiments may provide more insight into this complex relationship.

\section{Acknowledgements}

V.B. thanks the valuable support of Ian Potter Foundation in establishing an Ian Potter NanoBioSensing Facility at RMIT University. Research funding support by the Australian Research Council in the form of a Future Fellowship to V.B. (FT140101285) and Linkage Grants to V.B. and R.S. (LP130100437) is also acknowledged. The authors acknowledge the facilities, and the scientific and technical assistance of the Australian Microscopy \& Microanalysis Research Facility at the RMIT Microscopy \& Microanalysis Facility.

\section{Notes and references}

1. J. Tang, F. Luan and X. Chen, Bioorganic \& Medicinal Chemistry, 2006, 14, 3210-3217.

2. T. Sen, S. Mandal, S. Haldar, K. Chattopadhyay and A. Patra, The Journal of Physical Chemistry C, $2011,115,24037-24044$.

3. T. Horbett, L. Brash John and W. Norde, eds., Proteins at Interfaces III State of the Art, American Chemical Society, 2012.

4. L. Fei and S. Perrett, International Journal of Molecular Sciences, 2009, 10, 646-655.

5. F. Ramezani and H. Rafii-Tabar, Molecular BioSystems, 2015, 11, 454-462.

6. C. Carnovale, G. Bryant, R. Shukla and V. Bansal, Progress in Materials Science, 2016, 83, 152-190.

7. T. Chatterjee, B. K. Chatterjee, T. Saha, K. M. Hoque and P. Chakrabarti, Biochimica et Biophysica Acta (BBA) - General Subjects, 2017, 1861, 977-986.

8. A. M. Alkilany and C. J. Murphy, Journal of Nanoparticle Research, 2010, 12, 2313-2333.

9. $\quad$ S. Link and M. A. El-Sayed, Annu. Rev. Phys. Chem., 2003, 54, 331-366.

10. K. L. Kelly, E. Coronado, L. L. Zhao and G. C. Schatz, The Journal of Physical Chemistry B, 2003, 107, 668-677.

11. S. Sangabathuni, R. Vasudeva Murthy, P. M. Chaudhary, M. Surve, A. Banerjee and R. Kikkeri, Nanoscale, $2016,8,12729-12735$.

12. X. Xie, J. Liao, X. Shao, Q. Li and Y. Lin, Scientific Reports, 2017, 7, 3827.

13. J. Yue, T. J. Feliciano, W. Li, A. Lee and T. W. Odom, Bioconjugate Chemistry, 2017, 28, 1791-1800.

14. F. Ramezani, M. Amanlou and H. Rafii-Tabar, J. Nanopart. Res., 2014, 16, 2512.

15. J. Gao, C. M. Bender and C. J. Murphy, Langmuir, 2003, 19, 9065-9070.

16. L. Polavarapu, D. Zanaga, T. Altantzis, S. Rodal-Cedeira, I. Pastoriza-Santos, J. Pérez-Juste, S. Bals and L. M. Liz-Marzán, Journal of the American Chemical Society, 2016, 138, 11453-11456. 
17. L. Minati, F. Benetti, A. Chiappini and G. Speranza, Colloids and Surfaces A: Physicochemical and Engineering Aspects, 2014, 441, 623-628.

18. E. E. Connor, J. Mwamuka, A. Gole, C. J. Murphy and M. D. Wyatt, Small, 2005, 1, 325-327.

19. S. H. Lacerda, J. J. Park, C. Meuse, D. Pristinski, M. L. Becker, A. Karim and J. F. Douglas, ACS nano, 2010, 4, 365-379.

20. L. Shang, Y. Wang, J. Jiang and S. Dong, Langmuir, 2007, 23, 2714-2721.

M. Iosin, F. Toderas, P. Baldeck and S. Astilean, Journal of Molecular Structure, 2009, 924, 196-200.

22. Y. Chen and M. D. Barkley, Biochemistry, 1998, 37, 9976-9982.

23. S. Sugio, A. Kashima, S. Mochizuki, M. Noda and K. Kobayashi, Protein engineering, 1999, 12, 439-446.

24. C. Leggio, L. Galantini and N. V. Pavel, Physical Chemistry Chemical Physics, 2008, 10, 6741-6750.

25. T. Peters, in All About Albumin, ed. T. Peters, Academic Press, San Diego, 1995, DOI: https://doi.org/10.1016/B978-0125521109/50004-0, pp. 9-II.

26.

G. Sudlow, D. Birkett and D. Wade, Molecular Pharmacology, 1975, 11, 824-832.

27.

G. Sudlow, D. Birkett and D. Wade, Molecular Pharmacology, 1976, 12, 1052-1061.

28.

I. Petitpas, A. A. Bhattacharya, S. Twine, M. East and S. Curry, Journal of Biological Chemistry, 2001, 276, 22804-22809.

29.

R. G. Rayavarapu, C. Ungureanu, P. Krystek, T. G. van Leeuwen and S. Manohar, Langmuir, 2010, 26, 5050-5055.

30.

M. Liu and P. Guyot-Sionnest, J Phys Chem B, 2005, 109, 22192-22200.

31.

B. Nikoobakht and M. A. El-Sayed, Chemistry of Materials, 2003, 15, 1957-1962.

32.

J. E. Millstone, W. Wei, M. R. Jones, H. Yoo and C. A. Mirkin, Nano Letters, 2008, 8, 2526-2529.

33.

M. Kim, H. J. Park, S. W. Han, J. Park and W. S. Yun, Bulletin of the Korean Chemical Society, 2013, 34, 2243-2244.

34.

A. Sułkowska, Journal of molecular structure, 2002, 614, 227-232.

35.

S. Link, M. Mohamed and M. El-Sayed, The Journal of Physical Chemistry B, 1999, 103, 3073-3077.

36.

J. E. Millstone, S. J. Hurst, G. S. Métraux, J. I. Cutler and C. A. Mirkin, Small, 2009, 5, 646-664.

37.

E. Ringe, M. R. Langille, K. Sohn, J. Zhang, J. Huang, C. A. Mirkin, R. P. Van Duyne and L. D. Marks, The journal of physical chemistry letters, 2012, 3, 1479-1483.

38.

J. R. Lakowicz and G. Weber, Biochemistry, 1973, 12, 4161-4170.

39.

O. Stern and M. Volmer, Physik Zeitschr, 1919, 20, 183-188.

40.

E. L. Gelamo, C. H. T. P. Silva, H. Imasato and M. Tabak, Biochimica et Biophysica Acta (BBA) - Protein Structure and Molecular Enzymology, 2002, 1594, 84-99.

41. W. Vaughn and G. Weber, Biochemistry, 1970, 9, 464-473.

42. C. J. Orendorff and C. J. Murphy, The Journal of Physical Chemistry B, 2006, 110, 3990-3994.

43. A. Chaudhary, A. Gupta, S. Khan and C. K. Nandi, Physical Chemistry Chemical Physics, 2014, 16, 20471-20482.

44. W. Zhang, Q. Zhang, F. Wang, L. Yuan, Z. Xu, F. Jiang and Y. Liu, Luminescence, 2015, 30, 397-404. 
45. G. W. C. Kaye and T. H. Laby, Tables of physical and chemical constants: and some mathematical functions, Longmans, Green and Company, 1921.

46. V. Vasumathi and J. Santhanalakshmi, 2014.

47. J. Mariam, P. Dongre and D. Kothari, Journal of fluorescence, 2011, 21, 2193-2199.

48. S. Chakraborty, P. Joshi, V. Shanker, Z. Ansari, S. P. Singh and P. Chakrabarti, Langmuir, 2011, 27, 7722-7731.

49. P. Joshi, S. Chakraborty, S. Dey, V. Shanker, Z. A. Ansari, S. P. Singh and P. Chakrabarti, Journal of Colloid and Interface Science, $2011,355,402-409$. 